\title{
Heat Walk: Robust Salient Segmentation of Non-rigid Shapes
}

\author{
William Benjamin, Andrew Wood Polk, S.V.N. Vishwanathan and Karthik Ramani \\ Purdue University , USA
}

\begin{abstract}
Segmenting three dimensional objects using properties of heat diffusion on meshes aim to produce salient results. The few existing algorithms based on heat diffusion do not use the full knowledge that can be gained from heat diffusion and are sensitive to varying kinds of perturbations. Our simple algorithm, Heat Walk, converts the implicit information in the heat kernel to explicit knowledge about the pathways for maximum heat flow capacity. We develop a two stage strategy for segmentation. In the first stage we quickly identify regions which are dominated by heat accumulators by employing a greedy algorithm. The second stage partitions out dissipative regions from the previously discovered accumulative regions by using a KL-divergence based criterion. The resulting algorithm is both independent of human intervention and fast because of the globally aware directed walk along the maximal heat flow capacity. Extensive experimental evidence shows the method is robust to a variety of noise factors including topological short circuits, surface holes, pose variations, variations in tessellation, missing features, scaling, as well as normal and shot noise. Comparison with the Princeton Segmentation Benchmark (PSB) shows that our method is comparable with state of the art segmentation methods and has additional advantages of being robust and self contained. Based upon theoretical insight the convergence and stability of the Heat Walk is shown.

Categories and Subject Descriptors (according to ACM CCS): I.3.5 [Computer Graphics]: Computational Geometry and Object Modeling-Geometric algorithms, languages, and systems
\end{abstract}

\section{Introduction}

The amount of three-dimensional shape data is exploding because of the widespread availability of data acquisition techniques such as laser scanners, medical scanning equipment and tessellation software. Reducing the complexity of operations in geometry processing requires the division of a shape into parts which, in turn, can be processed separately and analyzed efficiently. Three-dimensional shape segmentation is therefore an important operation which has direct applications in diverse fields like shape search and matching [BBGO11], skeletonization [KT03] , texture mapping [LPRM02], and mesh simplification [CSAD04]. While segmentation itself is a well studied problem in many domains including machine learning and data mining, there are very few methods that can exploit the intrinsic structure of the three-dimensional data to discover meaningful segments. The goal of our research is to discover the inherent structure of the shape without prior knowledge of the number of segments or seed points.

Overview of our method: Given a three-dimensional shape approximated by a mesh $M$, we can represent it as a graph
$G(V, E)$ with vertex set $V$ and edge set $E$. Our objective is to decompose this graph into salient segments. One can best understand our model by drawing an analogy to the watershed model: when the water flows from the hills in the direction of least resistance. However, each individual drop only needs local information in order to decide the direction of its flow. The collective effect of these local decisions is that a large volume of water accumulates in the basins of attraction. In the watershed model the flow of water is analogous to heat flow. The steps in our segmentation pipeline are as follows (also See Figure 1):

1. Heat flow mapping: This step generates a heat distribution function on the mesh $M$ based on the curvature and connectivity of the points. In terms of the analogy, this step determines the topography of the paths of the water flow from the hills to the basins.

2. Heat walk based accumulator region identification: In this step we trace the paths from each point to the closest points of accumulation of heat. Accumulation regions are classified as the regions in which all points trace their paths to the same termination point. This is analo- 

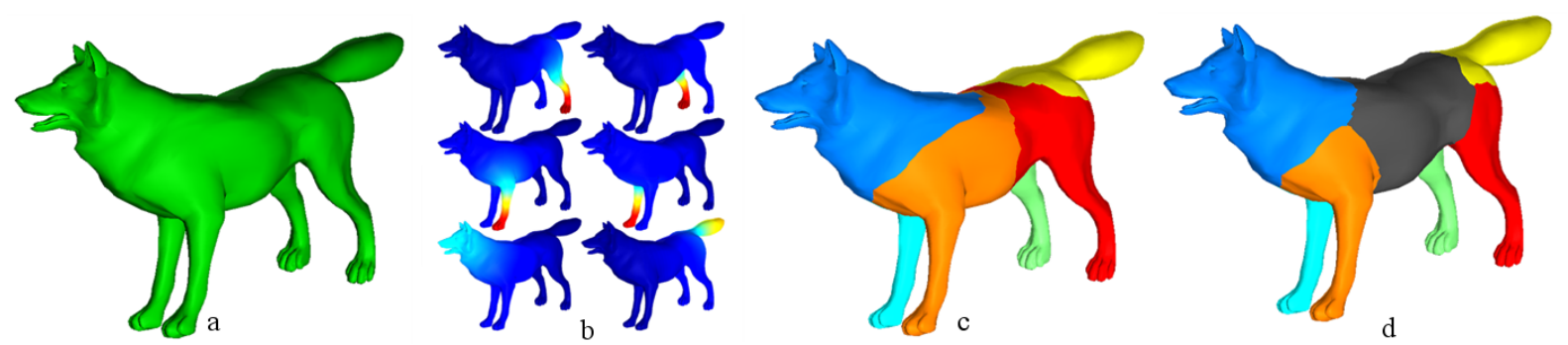

Figure 1: Pipeline of segmentation a) The input model b) Heat Flow mapping.The heat kernel is computed for the model c) The Heat Walk based accumulator region identification d) Relative Entropy based dissipator region identification

gous to the process of tracing the flow of the water along the streams to basins of accumulation. The regions of land whose drops ended in a particular basin are assigned to the basin. The basins emerge on-the-fly based on the topology of the mesh and without any prior knowledge or user input.

3. Relative entropy based dissipator regions identification: Once the heat accumulator regions are identified, we perform binary classification to determine if a point is a part of an accumulator or dissipator region. This is done depending on how close each point is to the uniform distribution of heat. This segments out the flatter regions of the three dimensional object. In terms of the analogy we discover the dissipator regions by how quicky they shed water.

Contributions The main contributions of our paper are:

1. We show that the heat kernel has implicit information about the shape in Section 3 and provide a method of converting the implicit information about the intrinsic structure of the shape in the heat kernel into explicit knowledge in terms of the segments of the shape.

2. In Section 4 we introduce a greedy algorithm called the Heat Walk which is globally aware and converges to the set of exemplars (representatives) for the model.

3. In Section 5 we characterize dissipative regions and describe a method of classifying regions into dissipative regions using a relative entropy method.

4. Our experiments in Section 6 provide a comprehensive evaluation of the method against a variety of perturbations.

\section{Related Work}

Mesh segmentation is a non-trivial problem and many methods have been proposed. However, there is a lack of consistency in understanding segmentation as a cognitive process. A recent benchmark paper on segmentation ameliorates this issue to an extent [CGF09]. A salient segment can be defined as the perceptually pertinent subset of the shape which draws or focuses the attention of the viewer to itself relative to its adjacent regions because of its relative size and degree of protrusion.

We define Robust Salient Segmentation (RSS) as a segmentation method that decomposes a model into salient segments while being robust to aberrations such as noise, shot noise, missing data in the form of holes and missing features, topological short circuits, pose variations, variations in tessellation and scaling. RSS is the overreaching goal of most segmentation methods. The reader is guided to two recent surveys by Attene et al. [AKM* 06, CGF09] and Shamir [Sha08] which provide an extensive overview of the available mesh segmentation methods.

Our method is related to watershed based methods [MW99, PKA03] which segment regions into catchment basins. These methods have very low tolerance to noise because local properties like principal curvatures are easily affected by noise. Spectral Methods like [LZ07] produce very good segmentations, they however use local properties like geodesic distances or curvatures which are affected by noise.

Complete adherence to the goal of RSS with no human intervention is a challenging problem because of requirement for an input parameter like $k$ [STK02] and sensitivity to local perturbations [LLS* 05,LZHM06, KT03, AFS06,LHMR08].

Recently diffusion based methods have shown promise in achieving the goal of RSS because they exploit the intrinsic geometry of the shape [SOG09, Gri06]. Our paper is closely related in spirit to diffusion based methods of segmentation hence we focus our attention only to these paramount methods.

Diffusion based methods alleviate the problem of pose variance [dGGV08, SOCG10, CMK ${ }^{*}$, GBAL09]. In [Rus07] the authors define GPS coordinates on the mesh which are clustered using a $k$-means algorithm to segment a shape. The segmentation achieved is salient and robust to short circuiting, however it needs human input in the form of $k$. Reuter et al. $\left[\mathrm{RBG}^{*} 09\right]$ on the other hand use the nodal sets of eigenvectors to segment a shape. The user makes a choice of $k$ eigenvectors to compose the desired segmentation. Reuter et al. [Reu10] use the persistence diagrams to construct heirarchical segmentation using eigenvectors of the Laplace Bel- 
trami operator while Goes et al. [dGGV08] partition shapes by iteratively refining segments from initial seeds. However they do not address the issue of robustness to all kinds of perturbations.

Two current heat kernel based methods which closely approach the goal of RSS without human intervention are Skraba et al. [SOCG10] and Dey et al. [DLL*10] They use persistence based clustering to identify feature points at local maxima of a vertex valued function, the Heat Kernel Signature [SOG09]. Vertex valued functions are relatively less stable than edge valued functions on a mesh to all sorts of local aberrations, specifically shot noise.

Our method also uses the spectrum of the LaplaceBeltrami operator and in addition we also extract more information to subsequently discover the intrinsic geometry without any assumption on $k$. In contrast to [SOCG10] and [DLL*10], we use an edge weighted operator which makes our method stable under high intensity of perturbations. Existing algorithms based on heat diffusion have not completely addressed the issues of robustness to all kinds of noise. We present a method that obtains RSS by exploiting the full information available from the heat kernel.

\section{Heat Flow and the Heat Kernel}

Let $\mathcal{M}$ be a Riemannian manifold, $\mu$ be a measure defined on $\mathcal{M}$, and let $L^{2}(\mathcal{M})$ (abbreviated as $L^{2}$ ) be the space of square integrable functions on $\mathcal{M}$. Heat diffusion on $\mathcal{M}$ is governed by the heat equation: $\Delta u(x, t)=-\frac{\partial u(x, t)}{\partial t}$ where $u(x, t)$ is the heat distribution at a point $x \in \mathcal{M}$ at time $t$, and $\Delta$ denotes the Laplace Beltrami Operator (LBO). Let $f: \mathcal{M} \rightarrow \mathbb{R}$ be a square integrable function which gives the initial distribution of heat on $\mathcal{M}$ at time 0 . The heat distribution at a subsequent time $t>0$ is denoted by $H_{t}(f)$. The so-called heat kernel $h_{t}: \mathcal{M} \times \mathcal{M} \rightarrow \mathbb{R}$ is the fundamental solution of the heat equation, that is, it satisfies for all $f \in L^{2}$, all $x \in \mathcal{M}$, $H_{t} f(x)=\int_{\mathcal{M}} h_{t}(x, y) f(y) d \mu(y)$.

The heat kernel controls the geometry-dependent propagation of heat across the shape. One can understand $h_{t}(x, y)$ as the quantity of heat that arrives at location $y$ after time interval $t$ if unit quantity of heat is inserted at location $x$. It is well known that the heat kernel can also be expressed in terms of the eigenvalues $\lambda_{i}$ and eigenvectors $\phi_{i}$ of the LBO [Gri06]:

$$
h_{t}(x, y)=\sum_{i} e^{-\lambda_{i} t} \phi_{i}(x) \phi_{i}(y) .
$$

Relation to Geodesic Distance On any weighted Manifold $(\mathcal{M}, \mu)$ of dimension $\eta$, there exists a smooth positive function $u(x, y)$ on $\mathcal{M} \times \mathcal{M}$ such that $\forall x \in \mathcal{M}$ we have $\lim _{t \rightarrow 0} \frac{1}{(4 \pi t)^{\eta / 2}} \exp \left(-\frac{d^{2}(x, y)}{4 t}\right) u(x, y)=h_{t}(x, y)$. Here $d^{2}(x, y)$ is the geodesic distance between the points $x$ and $y$ [Gri06]. Intuitively this means that for small values of $t$ the heat kernel can be approximated by a small geodesic neighbourhood of point $x$. We define the diffusion radius of a point as the geodesic distance from the point, within which $90 \%$ of the heat is accumulated. Intuitively, regions that accumulate heat will have a smaller diffusion radius as compared to regions that dissipate heat.

Relation to Curvature: The heat kernel is related to the curvature of $\mathcal{M}$. The diagonal values of the heat kernel are given by $h_{t}(x, x)=(4 \pi t)^{-\eta / 2} \sum_{i} a_{i} t^{i}$ where $a_{0}=1$ and $a_{1}=$ $\frac{1}{6} s(x)$ where $s(x)$ is the scalar curvature at point $x$ [MP49].

Points of high curvature have a tendency to attract heat. Thus over a period of time heat tends to accumulate in these regions. In other words, these points are very slow at dissipating heat compared to the rate at which they receive it. We call such points accumulators. On the other hand, the flatter regions tend to dissipate heat faster than they receive it. The heat distribution around such points will nearly be uniform. We call such points dissipators. (See Figure 2) In real

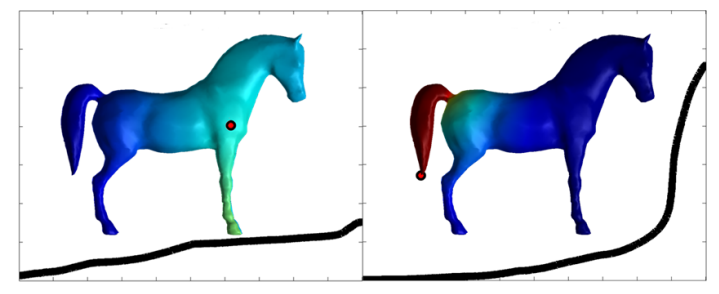

Figure 2: Comparison of heat distribution at the same time $t$ for a dissipator (left) and accumulator (right), both marked with a red dot. The distribution of heat in the left figure is comparatively more uniform than the heat distribution in the right.

life, instead of a smooth continuous manifold $\mathcal{M}$, we have to deal with a finite representation of an object as a mesh $M$. One can think of the mesh as a graph $G(V, E)$. In order to calculate the heat kernel on $M$ we need to compute the LBO on the mesh. The LBO on discrete meshes has been studied extensively and various discretizations have been proposed. The LBO on a surface is related to the mean curvature normal [Xu04], therefore many discretizations try to approximate this quantity. These schemes converge to the LBO in the limit while retaining some but not all of the key structural properties inherent to the continuous setting. We use the scheme used by Desbrun et al. [DMSB99] which is one of the faithful representations of the continuous LBO [WMKG08].

In order to define accumulators and dissipators on the finite mesh we work with $p_{x}(y)=h_{t}(x, y) / \sum_{y} h_{t}(x, y)$, the probability density function of the heat distribution at point $x$ and write:

Ideal Accumulator: The ideal accumulator is a point which retains all heat to itself. The probability density function of 
the heat distribution is given by

$$
p_{x}(y)=\delta_{x}(y)
$$

where $\delta_{x}(y)$ is an indicator function which takes on the value 1 when $x=y$ and 0 otherwise.

Ideal Dissipator: The ideal dissipator would be a point which distributes heat uniformly over all $N=|V|$ points on the mesh. The probability density function of the heat distribution in this case is given by

$$
p_{x}(y)=1 / N \forall y \in V
$$

In the sequel we present a method for identifying heat accumulator points on the shape while simultaneously assigning regions to those points.

\section{Heat Walk for Identifying Accumulators}

The goal of this step is to segment the model into accumulator regions which are sufficiently far apart from each other. We accomplish this by allowing each point to select a representative point called an exemplar. The points that choose the same exemplar are aggregated into the same accumulator region. The regions are merged in subsequent steps until the process terminates. We call this process the Heat Walk. A given point will be an exemplar for another point if two conditions are satisfied 1) The heat flow between the two points, as given by the heat kernel, is large, and 2) The exemplar itself has a strong tendency to attract heat. In other words it is an accumulator. Thus the choice of exemplar is a mutual voting process between the points and the prospective exemplars. The ideas mentioned here are formalized in following paragraphs.

Heat Potential: The tendency of a point to attract heat is expressed formally in terms of its heat potential $s^{m}(x)$. The heat potential is initialized as the diagonal values of the heat kernel $s^{1}(x)=h_{t}(x, x)$ and subsequent values are updated from previous values via

$$
s^{m+1}(x)=\max _{y \in \mathcal{E}}\left\{\min \left(h_{t}(x, y), s^{m}(y)\right)\right\}
$$

where $m$ is the count of the steps and $\mathcal{E}$ is the set of exemplars, initialized as $\mathcal{E}=V$.

Mutual voting process to choose an exemplar: The exemplar of a point $x$ is the point $y$ which maximizes the function $\min \left(h_{t}(x, y), s^{m}(y)\right)$. The mutual voting process described earlier is formalized by

$$
e^{m+1}(x)=\underset{y \in \mathcal{E}}{\operatorname{argmax}}\left\{\min \left(h_{t}(x, y), s^{m}(y)\right)\right\}
$$

where $e^{m+1}(x)$ is the exemplar for point $x$.

Pruning and Termination: The points which were not assigned as exemplars to any other point are excluded from subsequent steps. $\mathcal{E}$ is updated as the unique values of $e^{m+1}$. Thus the exemplar at a given step chooses an exemplar for itself in a subsequent step from the pool of existing exemplars $\mathcal{E}$. The accumulator regions pointing to an exemplar are merged into the accumulator region of the new exemplar. The process terminates when each exemplar chooses itself as its own exemplar, that is, the set of exemplars does not change between two iterations.

Figure 3 shows the incremental steps of the Heat Walk, at the far left all points are initialized to individual groups, moving right the primary accumulator regions have already formed and by the second step $75 \%$ of the points have been grouped into what will be their final grouping. The prominent features of the shape correspond to the accumulator regions. One can see that the smaller clusters gradually merge into the more stable accumulator regions. The pseudocode for the Heat Walk is given in the appendix as algorithm 1.

\subsection{Theoretical Remarks}

Now we shall show how the Heat Walk, mentioned previously, is closely connected to a random walk. Towards this end, we define a semiring $\mathcal{R}(H, \oplus, \otimes)$ over the set of values, $H$, generated by the heat kernel distribution $h_{t}$ together with the binary operations of $\max (\oplus)$ and $\min (\otimes)$. The internal laws are defined as $\forall\{a, b\} \in H, a \oplus b=\max (a, b)$, $a \otimes b=\min (a, b)$.The set of all $n$-tuples ( $n$-vectors ) over $\mathcal{R}$ is denoted by $\mathcal{R}_{n}$ and the set of all square matrices of order $n$ is given by $\mathcal{R}_{n \times n}$. Then Equation 4 can be written as a matrix vector product of $h_{t} \in \mathcal{R}_{n \times n}$ and $s^{m} \in \mathcal{R}_{n}$ in the new semiring

$$
s^{m+1}=h_{t} \otimes s^{m}
$$

where $s^{m+1}(x)=\oplus_{y \in \mathcal{E}} h_{t}(x, y) \otimes s^{m}(y)$. This formulation is analogous to a random walk in the usual semiring $R(H,+, \times)$ which is defined via $s^{m+1}=h_{t} s^{m}$. Instead of being a random walk, this process is a directed walk, which we call the Heat Walk.

Flow capacity is an idea from max-min algebra to find the bottlenecks in a path. We define the heat flow capacity $c(x, y)$ between a point $x$ and its possible exemplar $y$ as $c(x, y)=$ $h_{t}(x, y) \otimes s^{m}(y)$.

The exemplar of a point is chosen along the greatest heat flow capacity. At every step of the Heat Walk, each point chooses an exemplar according to Equation 5 which can be rewritten as

$$
e^{m+1}(x)=\underset{y \in \mathcal{E}}{\operatorname{argmax}}\{c(x, y)\}
$$

We can see that the exemplar for a point $x$ is chosen as the point $y$ that maximizes $c(x, y)$. The heat potential at a point $x$ is then updated as the maximum capacity.

Convergence: The Heat Walk converges when $e^{m}(x)=$ $e^{m-1}(x)$ for all $x$, that is, the exemplars in the previous state are the same as the exemplars in the present state.

Path: The path of the Heat Walk from a point $x$ is the 


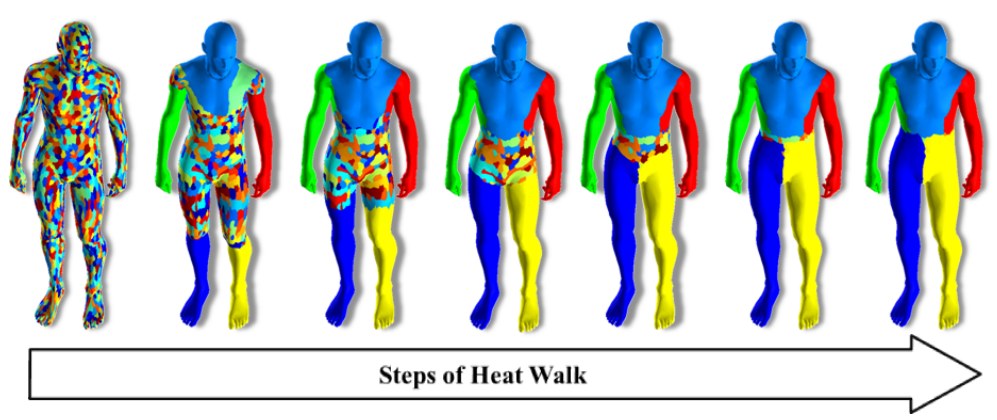

Figure 3: This figure demonstrates that the Heat Walk converges very quickly to the dominant accumulator regions.

ordered sequence $\pi_{x}=\left\{x, e^{1}(x), e^{2}\left(e^{1}(x)\right) \ldots\right\}$ of a point and its exemplars in the subsequent states.

Optimality of the path: The Heat Walk proceeds along the path of maximal flow capacity. At every step of the Heat Walk each point chooses an exemplar. Each step of the walk is along the maximal flow capacity, thus the entire path from a point $x$ is along the path of maximal flow capacity.

The Heat Walk generates a tree: Each path from a point $x$ can be traced to its final exemplar. Going backwards from the final exemplar to the points we can trace a tree with the final exemplar at the root and the points as the leaves. From our observation these trees represent separate regions of the shape.

Finite Set Reduction: The set of heat flow values on the mesh is finite. The $(\oplus, \otimes)$ operations cannot introduce a new number to this set. Additionally the pruning operation reduces the number of elements in the set.

Convergence of Heat Walk: The Heat Walk applied to a finite set must converge. We obtain convergence when the heat walk returns the same exemplars.

Stability: Stability means that the effects of noise on the outcome are mitigated. Assume a normal distribution of noise on the surface of the mesh which perturbs $h_{t}(x, y)$ and $s^{m}$. The min operation $\otimes$ tends to filters out high values of noise, Then the max operation $\oplus$ tends to filter low values of noise, leaving only a narrow band of noise. Thus the effective error is mitigated. Additionally, pruning removes nonexemplars adding to the stability.

\section{Identifying Dissipator Regions}

After having classified the points into accumulator regions in section 4, the next step is to discover the dissipator regions. Dissipative regions correspond to relatively featureless regions. We revisit the idea of the ideal heat dissipators and accumulators and develop a strategy for classification using the celebrated Kullback-Liebler divergence (KL Divergence) for comparing distributions [CT91]. Recall that the
KL divergence between distributions $p$ and $q$ is given by

$$
D_{K L}(p \| q)=\sum_{y} p(y) \log \frac{p(y)}{q(y)}
$$

This is the measure of additional amount of information to specify $p$ in terms of $q$. KL-divergence is a non negative quantity $D_{K L}(p \| q) \geq 0$, with equality if and only if the distributions $p$ and $q$ are identical. Furthermore, it has the property that it is resistant to local noise because the probability mass of the heat distribution is relatively invariant to local perturbation.

An ideal dissipator has a uniform heat distribution. In order for a point to belong to the dissipator region, it must be less divergent from the uniform heat distribution. On the other hand, we use the mean of the heat distribution of the segment as a criteria for determining the divergence of a point from the accumulator region. Separation of the dissipative region begins by computing the divergence of each points' probability density function $p_{x}(y)=\frac{h_{t}(x, y)}{\sum_{y} h_{t}(x, y)}$ with respect to first the uniform density $\mathrm{Eq}(3)$ and later to its respective mean cluster density $p_{C}(y)=\frac{\sum h_{x \in C}(x, y)}{\sum_{x \in C} \sum_{y} h_{t}(x, y)}$ where $\mathrm{C}$ is the cluster. The divergences are compared and when the

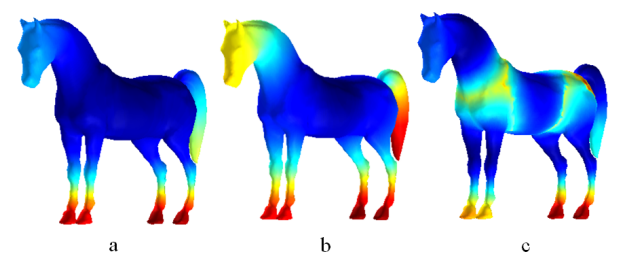

Figure 4: a) The HKS function $b$ ) The KL divergence of the points from the uniform distribution. c) KL divergence of the points from the cluster mean

divergence from the uniform is less than that from the mean of the cluster the point will be reclassified as a member of the dissipative region. The pseudocode for this is given in the appendix as algorithm 2.

Figure 4 shows 3 different functions on the mesh. Fig- 
ure $4 \mathrm{a}$ and $\mathrm{b}$ provide a contrast between the Heat Kernel Signature (HKS) and the KL divergence from the uniform distribution. The KL divergence on the head of the horse is more informative compared to the HKS and Fig $4 \mathrm{c}$ plots the KL divergence from the cluster mean of the segments.

\section{Experiments}

We demonstrate the stability of our method and insensitivity to the type of mesh employed, pose, noise, scale and topological short circuits. The three-dimensional models used in our experiments are taken from the PSB [CGF09], as well as the Tools for non-rigid Shape Comparison and Analysis (TOSCA) database [BBK08].

\subsection{Versatility}

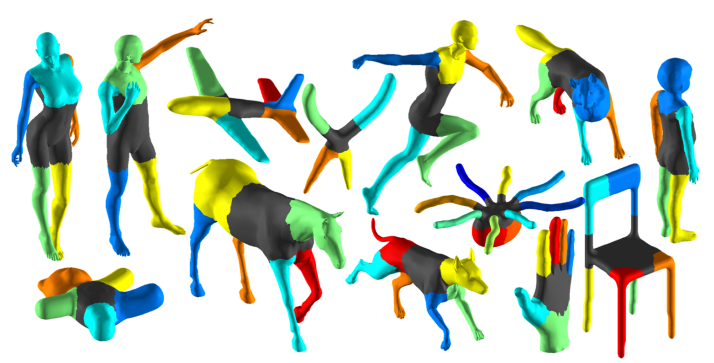

Figure 5: Segmentation results across wide varieties of models. Further results at https://engineering.purdue.edu/precise/heatwal

\begin{tabular}{|r|c|c|c|r|r|}
\hline Method & CD & RI & HD & \multicolumn{2}{|c|}{ CE } \\
\hline & & & & 1 & 2 \\
\hline Humans & 0.140 & 0.081 & 0.108 & 0.082 & 0.055 \\
\hline HeatWalk & 0.267 & 0.148 & 0.234 & 0.221 & 0.136 \\
\hline RandCuts & 0.150 & 0.093 & 0.127 & 0.149 & 0.083 \\
\hline ShapeDiam & 0.221 & 0.143 & 0.177 & 0.152 & 0.095 \\
\hline CoreExtra & 0.272 & 0.159 & 0.177 & 0.144 & 0.094 \\
\hline RandWalks & 0.297 & 0.164 & 0.215 & 0.222 & 0.123 \\
\hline FitPrim & 0.253 & 0.145 & 0.249 & 0.265 & 0.174 \\
\hline Kmeans & 0.288 & 0.161 & 0.268 & 0.286 & 0.190 \\
\hline
\end{tabular}

Table 1: Comparison of results of Heat Walk based segmentation of non-rigid models against segmenation based on Humans [CGF09], Randomized cuts [GF08], Shape Diameter [SSCO08], Core extraction [KLT05], Random Walks [LHMR08], Fitting primitives [AFS06] and k-Means [STK02].The metrics used are Cut Discrepancy(CD), Hamming Distance (HD), Rand Index (RI) and Consistency Error $(C E)$



Figure 6: Segmentation for various poses of the cat and gorilla model
Our algorithm is versatile and is able to produce a visually pleasing segmentation across a wide range of models such as humans, animals, toys, furniture, and tools as demonstrated in Figure 5. The results show that the method is more applicable to nonrigid models like humans, animals etc because they have strong accumulator regions. Table 1 shows the results of the Heat Walk segmentation compared to available methods from the benchmark [CGF09] across eleven categories of non-rigid three dimensional models. Please refer to [CGF09] for details of the metrics. The results show that the Heat Walk is comparable in performance to the majority of the methods presented in the benchmark. We do not do any cut refinement like [GF08, SSCO08, LHMR08, KLT05], which would improve the quality of the results. While the PSB serves the purpose for VSS validation, our primary aim is to study RSS using Heat Walk. The next few sections confirm the robustness of the method using models with added noise that are not available in PSB.

\subsection{Consistency}

Next we show that our method is insensitive to pose and intra class variations in the models (See Figure 6). As can be seen, the gross characteristics of the segmentation for a given model are stable across the different poses. The cuts appear consistently along the joints of the heads and legs of the gorilla and along the tail, head, and legs of the cat models. Pose invariance of the Heat Walk is caused by the isometry invariance of the heat kernel. We are also interested in

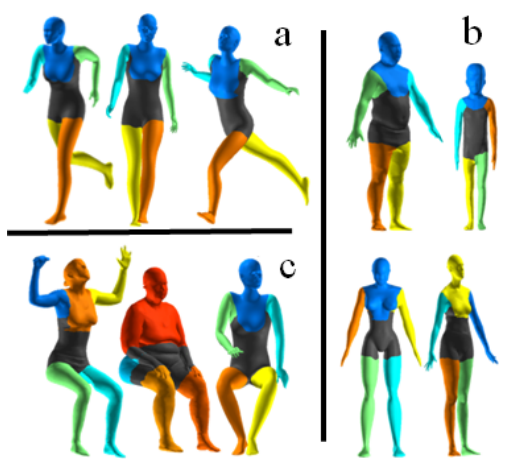

Figure 7: Segmentation for human models: a) articulations of the same model $b$ ) within the same class $c$ ) bending near the joint of the legs and merging of the hands into the body.

consistency across the same class of models (See Figure 7 
a). The models in Figure $7 \mathrm{~b}$ shows consistency for different models of the human class of models. Figure $7 \mathrm{c}$ shows the results for the sitting pose of human models. Our method is unable to identify the arms and head separately in the central model because the arms are completely fused with the body. The heat distribution in this case is drastically changed when compared to a clean model.

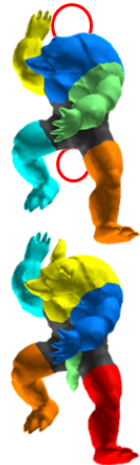

a

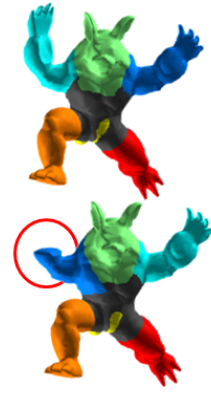

b

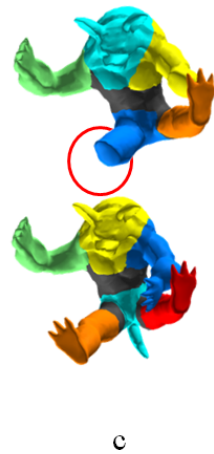

Figure 8: Segmentation is resistant to missing parts (red circles).

\begin{tabular}{|r|r|r|r|r|r|}
\hline \multicolumn{7}{|c|}{ short circuits } \\
\hline \# Shorts & 10 & & & & \\
\hline $\mathrm{CP}(\%)$ & 2.32 & & & & \\
\hline \multicolumn{7}{|c|}{ Holes } \\
\hline \# Holes & 58 & 106 & 154 & 202 & 250 \\
\hline $\mathrm{CP}(\%)$ & 1.23 & 1.89 & 2.39 & 3.08 & 3.39 \\
\hline \multicolumn{7}{|c|}{ Noise } \\
\hline \% Noise & 6 & 17 & 34 & 46 & 63 \\
\hline $\mathrm{CP}(\%)$ & 1.51 & 2.38 & 3.9 & 5.02 & 6.1 \\
\hline \multicolumn{7}{|c|}{ Shot Noise } \\
\hline \% Perturbed & 5.88 & 17.65 & 29.41 & 47.06 & 52.94 \\
\hline CP(\%) & 3.61 & 2.97 & 5.07 & 6.75 & 7.28 \\
\hline
\end{tabular}

Table 2: Change Percentage $(\mathrm{CP})$ is a consistency measure which tells twice the percentage of points in the original segmentation that must change their segment membership to become the new segmentation. The consistency of segmentation under various kinds of perturbations is high.

Figure $8 \mathrm{a}, \mathrm{b}$ and $\mathrm{c}$ brings out the consistency between complete and incomplete models. The resulting segmentation follows the overall theme of segmentation of the complete models. Table 2 shows that our method is consistent under noise using a consistency metric.

\subsection{Resistance to Random Noise}

We apply increasing intensity of random normal noise to the models (See Figure 9). Noise was added to all the points in the direction of the normal at the point. The noise values shown on the top of each hand model in Fig 9 gradually increase from 0 to $10 \%$ of the diagonal of the bounding box.

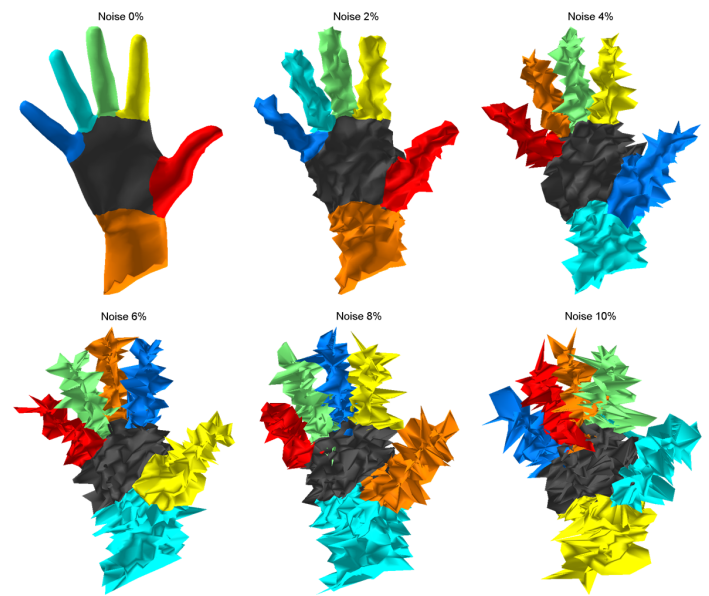

Figure 9: Segmentation results on noise corrupted models. The value at the top of each figure corresponds to the fraction of noise added.

The cuts corresponding to the fingers of the hand are largely insensitive to noise. Up to $10 \%$ noise our method performs well. At very high noise levels salience of the segments according to cognition studies is lost, so consistency at large noise values $>10 \%$ is not pertinent.

\subsection{Shot Noise}

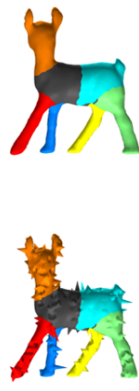

a

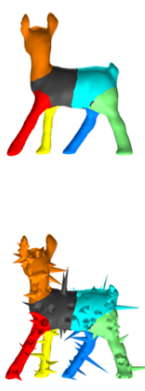

b

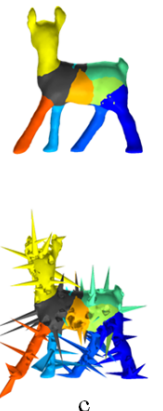



e
Figure 10: Segmenation is robust to shot noise. a,b,c,d,e show models addition of $11 \%, 23 \%, 40 \%, 51 \%$ and $63 \%$ noise to $6 \%$ of the points at the bottom with the results mapped to the original model at the top.

Shot noise is defined as corruption of three-dimensional data at finitely many points on the mesh which appear as spikes. We added shot noise to $6 \%$ of the points selected randomly on the model. The shot noise creates local accumulators which can change the heat flow. The images in Figure 10 show the results of increasing amounts of shot noise. The image on the top shows the results of the segmentation mapped to the original model. Shot noise in increasing intensity from $10 \%$ up to $80 \%$ of the model's bounding box di- 
agonal was applied. The results are consistent up to $23 \%$ of noise. The segmentation of the strong accumulator regions is consistent for a higher amount of noise. The Heat Walk has higher resistance to shot noise because the max min operator decimates any isolated perturbance.

\subsection{Resistance to Topological Noise and Short Circuits}

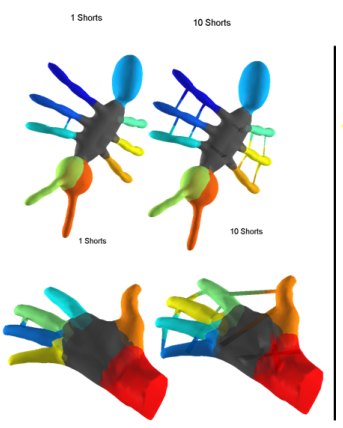

a

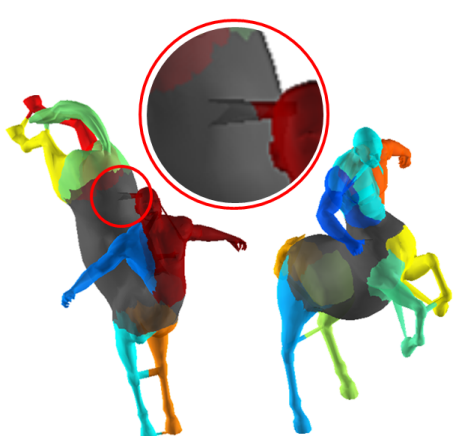

b
Figure 11: Topological short circuits on the ant, hand and centaur.

We generated models with increasing levels of short circuits ranging from 1 to 10 . The short circuits generated were located by sampling pairs of points which are close in terms of Euclidean distance but far apart geodesically. The segmentation results are robust to large numbers of short circuits. Figure 11 a shows the hand and the ant models which have been short circuited. The total number of short circuits is 1 on the left side and 10 on the right side. We have increased the transparency to show some short circuits which are internal. Figure $11 \mathrm{~b}$ shows two poses of the centaur model. The model on the right is well segmented into salient components. On the left the method fails when a weak accumulator region of the centaur model is short circuited with a strong dissipative region. Therefore the heat flow is diverted to the dissipative regions weakening the accumulative region hence the head merges with the arm. The robustness is due to aggregation of paths by the heat kernel and also due to the directed walk.

The second part of this experiment was to create holes in the models changing the heat flow on the surface. This was done due to our interest in testing the limit to which the segmentation is consistent with that of the original models. We randomly sampled points and removed the corresponding triangles creating holes on the surface. The number of triangles removed was $5 \%, 10 \%, 15 \%$, and $20 \%$ of the original triangles in the model. The segmentation was consistent with up to $15 \%$ of the triangles removed, after which the regions are hardly connected and the disconnected regions greatly affect the heat flow.(See Figure 12.) The Heat Walk is robust to missing triangles since the heat kernel between two points is the exponential sum of all possible paths of length

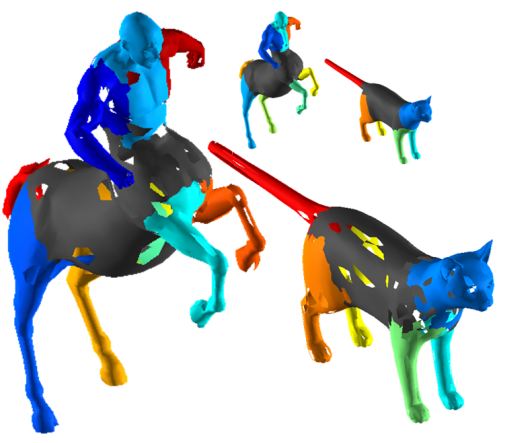

Figure 12: Models(large) with 15\% of the triangles removed are still consistent with the models without any missing triangles (small).

$\mathrm{t}$ between the two points. Removing some of the paths does not substantially change the heat flow.

\subsection{Sensitivity to tessellation and scale}

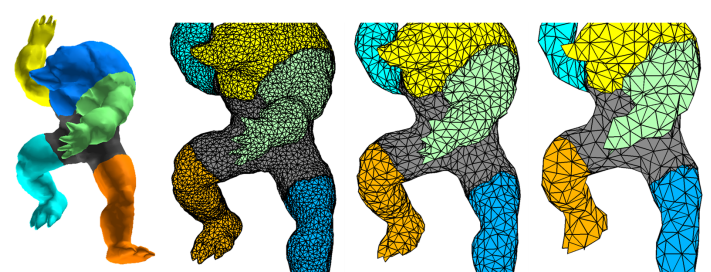

Figure 13: Sensitivity to tessellation. The Armadillo models with different qualities of meshes high, medium and low. The segmentation is consistent across different tessellations of the model.

We decimated the armadillo model down to several levels of tessellation and observed the behavior of the method. While the placement of the cuts may vary slightly, the method by and large recovers the same segmentation as can be seen in Figure 13. We scaled the ant model keep-

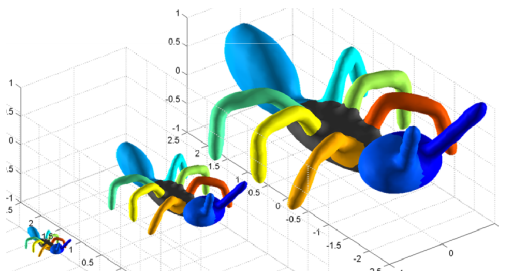

Figure 14: Results of the ant model scaled by $\times 0.5, \times 1.5$ and $\times 3$.

ing all other aspects of the model constant (See Figure 14 ). The segmentation was completely invariant to scale for 
$\times 0.5, \times 1.5, \times 3$ and $\times 10$ (not shown). Invariance to tessellation and scale is a result of the choice of the discretization of the LBO.

\subsection{Time}

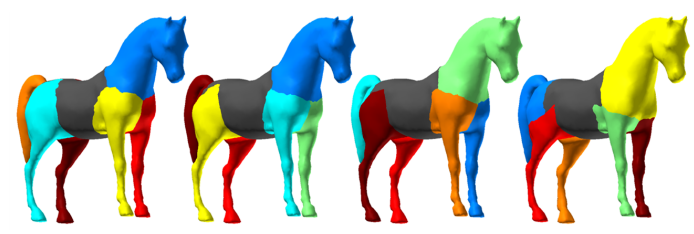

Figure 15: Segmentation over a range of times, from left to right $t=0.65, t=1.1, t=1.6$, and $t=3.9$.

In this section we explore the the stopping time for heat diffusion, in order to justify our assumption of time $t=1$ throughout all other experiments.Figure 15 shows the choice of $t=1$ is within a wide range of times that return similar segmentation. In a further experiment a sample of 100 models was taken at random and segmented at different times logarithmically spaced between $t=0.13$ and 12 . We found that for $71 \%$ of the models the number of segments did not change for time between 0.6 to 2.7. Over a smaller interval between 0.85 and $1.9,82.3 \%$ did not change. While there is no single value of time that can be used to described all shapes, from this experiment it is clear we have chosen a time that correlates to the global scale and is suitable for most models. Throughout all experiments the heat kernel was calculated for $t=1$,

\section{Limitations}

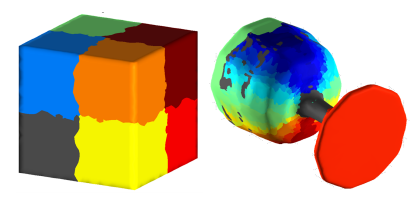

Figure 16: At left Heat Walk is able to identify 8 corners as strong accumulator regions. This is different from the intended segmentation into 6 surfaces. The cylindrical surface of a glass is oversegmented

When surface characteristics are more appropriate than salience for segmentation our method will be inappropriate (See Figure 16). Rigid objects like CAD models may not contain salient regions resulting in incorrect segmentation. For objects like a glass or a cylinder which lack dominant protrusions, the heat walk will oversegment. When the dissipative regions are disconnected with low heat affinities between each other, binary classification of the dissipative regions using KL divergence may fail.This may be corrected by modifying the magnitude of the uniform heat distribution.

\section{Conclusions and Future Work}

The Heat Walk algorithm partitions the shape in an unsupervised manner because it is data-driven while discovering the inherent number of segments. The method does not require choice of initial seed points and converges in a few iterations. The strategy of segmenting into accumulative and dissipative regions is simple and works well for non-rigid objects. Evaluation against state-of-the art methods available in the PSB show that the method is comparable without cut refinement. We have tested the limits of the algorithm for normal noise, shot noise, tessellation, topological noise, topological short circuits, scale, changes in pose and missing features. Our results show that the method is robust. This is because of the error mitigation by the max min operator and pruning at every step of the Heat Walk and also the insensitivity of KL Divergence to noise. Although the choice of time is suitable for most models, in the future work we would like to derive model specific optimal times. Additionally, exploiting local times for cut refinement will further improve the quality of segmentation.

\section{References}

[AFS06] Attene M., Falcidieno B., Spagnuolo M.: Hierarchical mesh segmentation based on fitting primitives. The Visual Computer 22 (2006), 181-193. 2, 6

[AKM*06] Attene M., Katz S., Mortara M., Patane G., SPAGNUOLO M., TAL A.: Mesh segmentation - a comparative study. In SMI '06: Proceedings of the IEEE International Conference on Shape Modeling and Applications 2006 (Washington, DC, USA, 2006), IEEE Computer Society, p. 7. 2

[BBGO11] Bronstein A. M., Bronstein M. M., Guibas L. J., Ovsjanikov M.: Shape google: Geometric words and expressions for invariant shape retrieval. ACM Trans. Graph. 30 (February 2011), 1:1-1:20. 1

[BBK08] Bronstein A., Bronstein M., Kimmel R.: Numerical geometry of non-rigid shapes. Springer (2008). 6

[CGF09] Chen X., Golovinskiy A., , Funkhouser T.: A benchmark for 3D mesh segmentation. ACM Transactions on Graphics (Proc. SIGGRAPH) 28, 3 (Aug. 2009). 2, 6

[CMK*] Cuzzolin F., Mateus D., Knossow D., Boyer E., HORAUD R.: Coherent laplacian 3-d protrusion segmentation. 2

[CSAD04] Cohen-Steiner D., Alliez P., Desbrun M.: Variational shape approximation. ACM Trans. Graph. 23 (August 2004), 905-914. 1

[CT91] Cover T. M., ThOMAs J. A.: Elements of information theory. Wiley-Interscience, New York, NY, USA, 1991. 5

[dGGV08] De Goes F., Goldenstein S., Velho L.: A hierarchical segmentation of articulated bodies. In SGP '08: Proceedings of the Symposium on Geometry Processing (Aire-laVille, Switzerland, Switzerland, 2008), Eurographics Association, pp. 1349-1356. 2, 3

[DLL*10] DEY T., LI K., LuO C., RANJAN P., SAFA I., WANG Y.: Persistent heat signature for pose-oblivious matching of incomplete models. Computer Graphics Forum 29, 5 (2010), 1545-1554. 3

[DMSB99] Desbrun M., Meyer M., Schröder P., BARR A. H.: Implicit fairing of irregular meshes using diffusion 
and curvature flow. In SIGGRAPH '99: Proceedings of the 26th annual conference on Computer graphics and interactive techniques (New York, NY, USA, 1999), ACM Press/AddisonWesley Publishing Co., pp. 317-324. 3

[GBAL09] Gębal K., Baerentzen J. A., Aanaes H., LARSEN R.: Shape analysis using the auto diffusion function. In Proceedings of the Symposium on Geometry Processing (2009), SGP '09. 2

[GF08] Golovinskiy A., Funkhouser T.: Randomized cuts for 3d mesh analysis. ACM Trans. Graph. 27, 5 (2008), 1-12. 6

[Gri06] GRIGOR'YAN A.: Heat kernels on weighted manifolds and applications. Cont. Math 398 (2006), 93-191. 2, 3

[KLT05] Katz S., Leifman G., TAL A.: Mesh segmentation using feature point and core extraction. The Visual Computer 21, 8-10 (2005), 649-658. 6

[KT03] KaTZ S., TAL A.: Hierarchical mesh decomposition using fuzzy clustering and cuts. In ACM SIGGRAPH 2003 Papers (New York, NY, USA, 2003), SIGGRAPH '03, ACM, pp. 954961. 1, 2

[LHMR08] LaI Y.-K., Hu S.-M., Martin R. R., Rosin P. L.: Fast mesh segmentation using random walks. In SPM '08: Proceedings of the 2008 ACM symposium on Solid and physical modeling (New York, NY, USA, 2008), ACM, pp. 183-191. 2, 6

[LLS*05] Lee Y., LeE S., Shamir A., Cohen-Or D., SeIDEL H.-P.: Mesh scissoring with minima rule and part salience. Comput. Aided Geom. Des. 22 (July 2005), 444-465. 2

[LPRM02] LÉVy B., PetitJean S., Ray N., MaIllot J.: Least squares conformal maps for automatic texture atlas generation. ACM Trans. Graph. 21 (July 2002), 362-371. 1

[LZ07] LIU R., ZHANG H.: Mesh segmentation via spectral embedding and contour analysis. Computer Graphics Forum 26, 3 (2007), 385-394. 2

[LZHM06] LAI Y.-K., ZhOU Q.-Y., HU S.-M., MARTIN R. R.: Feature sensitive mesh segmentation. In Proceedings of the 2006 ACM symposium on Solid and physical modeling (New York, NY, USA, 2006), SPM '06, ACM, pp. 17-25. 2

[MP49] Minakshisundaram S., Pleijel A.: Some properties of the eigenfuncions of the laplace operator on riemannian manifolds. Can. J. Math (1949). 3

[MW99] Mangan A. P., Whitaker R. T.: Partitioning 3d surface meshes using watershed segmentation. IEEE Transactions on Visualization and Computer Graphics 5 (October 1999), 308321. 2

[PKA03] Page D. L., Koschan A. F., Abidi M. A.: Perception-based $3 \mathrm{~d}$ triangle mesh segmentation using fast marching watersheds. Computer Vision and Pattern Recognition, IEEE Computer Society Conference on 2 (2003), 27. 2

[RBG*09] Reuter M., Biasotti S., Giorgi D., PATANÏ£; G., Spagnuolo M.: Discrete laplace-beltrami operators for shape analysis and segmentation. Computers \& Graphics 33, 3 (2009), 381 - 390. IEEE International Conference on Shape Modelling and Applications 2009. 2

[Reu10] REUTER M.: Hierarchical shape segmentation and registration via topological features of laplace-beltrami eigenfunctions. Int. J. Comput. Vision 89 (September 2010), 287-308. 2

[Rus07] RUSTAMOV R. M.: Laplace-beltrami eigenfunctions for deformation invariant shape representation. In SGP '07: Proceedings of the fifth Eurographics symposium on Geometry processing (Aire-la-Ville, Switzerland, Switzerland, 2007), Eurographics Association, pp. 225-233. 2
[Sha08] SHAmIR A.: A survey on mesh segmentation techniques. Comput. Graph. Forum 27, 6 (2008), 1539-1556. 2

[SOCG10] Skraba P., Ovsjanikov M., Chazal F., Guibas L.: Persistence-based segmentation of deformable shapes. In CVPR Workshop on Non-Rigid Shape Analysis and Deformable Image Alignment (June 2010), p. to appear. 2, 3

[SOG09] Sun J., Ovsjanikov M., Guibas L.: A concise and provably informative multi-scale signature based on heat diffusion. Computer Graphics Forum 28, 5 (2009), 1383-1392. 2,

[SSCO08] ShapiRa L., Shamir A., COHEn-Or D.: Consistent mesh partitioning and skeletonisation using the shape diameter function. The Visual Computer 24, 4 (2008), 249-259. 6

[STK02] Shlafman S., TAL A., KATZ S.: Metamorphosis of polyhedral surfaces using decomposition. In Computer Graphics Forum (2002), pp. 219-228. 2, 6

[WMKG08] WARDETZKY M., MATHUR S., KÄLBerer F. GRINSPUN E.: Discrete laplace operators: no free lunch. In $A C M$ SIGGRAPH ASIA 2008 courses (New York, NY, USA, 2008), SIGGRAPH Asia '08, ACM, pp. 19:1-19:5. 3

[Xu04] XU G.: Discrete laplace-beltrami operators and their convergence. Comput. Aided Geom. Des. 21, 8 (2004), 767-784. 3
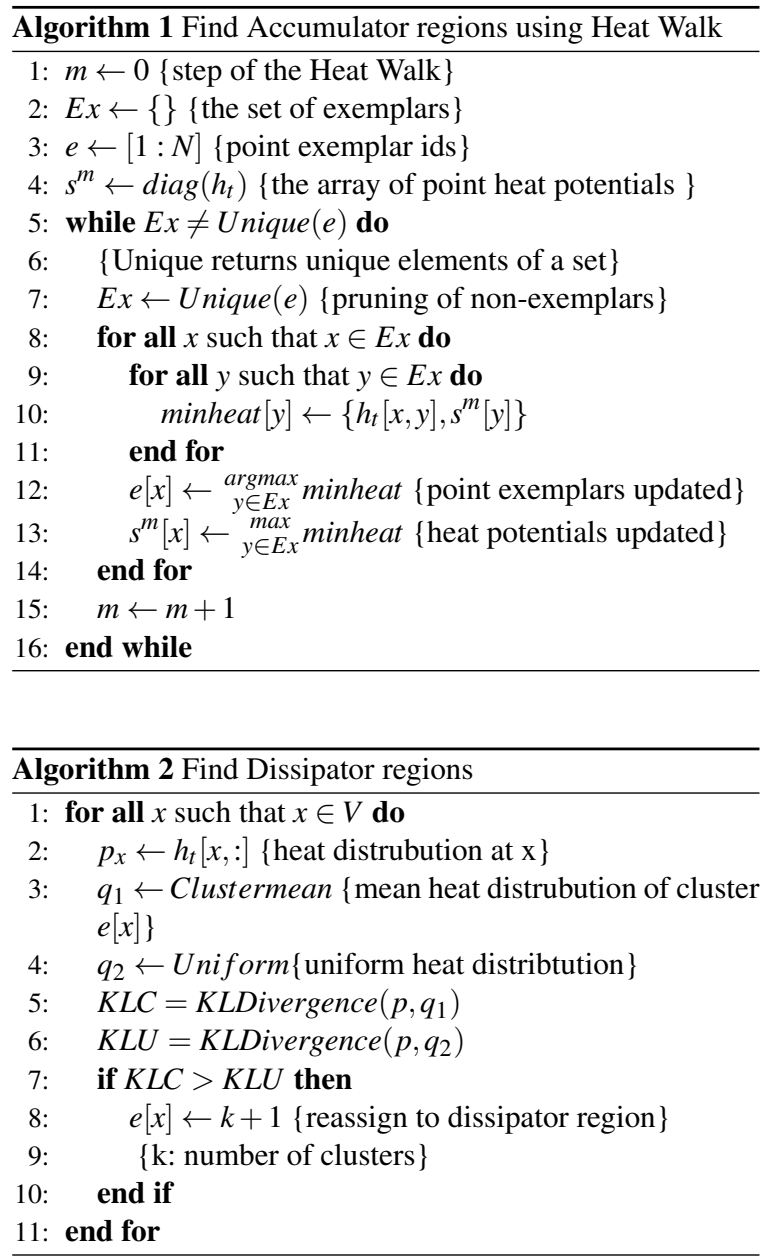